\title{
Observer Design for a Class of Nonlinear Systems Subject to Unknown Inputs *
}

\author{
Saleh Sayyaddelshad*, Thomas Gustafsson ** \\ * Control Engineering Group, Luleå University of Technology, \\ Luleå, 971 87, Sweden (e-mail: saleh.s.delshad@ltu.se). \\ ** Control Engineering Group, Luleå University of Technology, \\ Luleå, 971 87, Sweden (e-mail: tgu@ltu.se).
}

\begin{abstract}
This is an extended abstract of the author's recent work where a novel method to design an observer for a class of nonlinear discrete-time systems with unknown inputs is presented. To make a long story short, several proofs for the provided theorems and also simulation results have been intentionally neglected. In particular, based on Lyapunov functional, we derive a sufficient condition for existence of the designed observer which requires solving a nonlinear matrix inequality. In light of linear algebra, the achieved condition will be formulated in terms of linear matrix inequality (LMI) that can be solved by popular methods easily. Compared to recent researches, the developed approach shows a significant better performance than other techniques previously published in literature and is less conservative in terms of Lipschitz constant.
\end{abstract}

Keywords: Observer Design, Nonlinear Lipschitz Systems, Unknown Inputs, Linear Matrix Inequalities

\section{INTRODUCTION}

Observer design for nonlinear systems subject to unknown inputs has been one of the fascinating topics in control field. Although a variety of techniques exist in the literature, there are still many discussions on the observer performance and conservativeness. Convergence of the estimated states to the real states in a shorter time while the used technique is less conservative against the lipschitz constant is a momentous issue of many researches where the researchers try to find either a new technique or extend existing techniques. See for example Allgower (2008); Phanomchoeng (2010); Zemouche (2013) and references therein.

In this paper, a discrete-time observer for a class of Lipschitz discrete-time systems with unknown input is presented where the designed observer shows better performance compared to some recent similar researches. Some comparisons between our method and similar researches such as Allgower (2008), Phanomchoeng (2010) and Zemouche (2013) are provided where our results show a significant enhance in both observer performance and conservativeness. Although there is a direct effect of unknown input on the system structure, no negative impact on the estimator performance is sensible. The simulation results will be analyzed in two different subsections in terms of performance and conservativeness. Comparison with some well-known papers in the literature reveals different merits of our proposed method.

\section{PRELIMINARIES AND PROBLEM STATEMENT}

Consider the following nonlinear discrete-time system subject to unknown input:

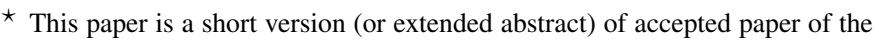
author which will be presented at the 13th European Control Conference (ECC) from the 24th to the 27th of June 2014 in Strasbourg, France.
}

$$
\begin{aligned}
x_{k+1} & =A x_{k}+B u_{k}+D f\left(x_{k}\right)+D_{1} \mu_{k}, \\
y_{k} & =C x_{k}
\end{aligned}
$$

where $x \in R^{n}, u \in R^{m}, \mu \in R^{q}$ and $y \in R^{p}$ are the state vector, known input, unknown input and output, respectively. Matrices $A, B, C, D$, and $D_{1}$ are real and with appropriate dimensions. The function $f$ is nonlinear and is assumed to be differentiable once. The aim is to design a nonlinear observer such that it can estimate the state vector $x_{k}$ in presence of unknown input $\mu_{k}$ asymptotically. It should be noted that $(A, C)$ is observable. To specify the class of nonlinear systems under study, we use the following assumption.

Assumption 1. We assume that the function $f\left(x_{k}\right)$ is locally Lipschitz with respect to $x$ in a region $\Upsilon$ containing the origin if $\|f(0)\|=0$ and,

$$
\|f(\alpha)-f(\beta)\| \leq \gamma\|\alpha-\beta\| \quad \forall \alpha, \beta \in \Omega
$$

where $\|$.$\| is the 2$-norm and $\gamma \geq 0$ is called the Lipschitz constant.

\section{MAIN RESULTS}

Consider the following nonlinear observer structure:

$$
\begin{aligned}
z_{k+1} & =N z_{k}+G u_{k}+L y_{k}+\operatorname{MDf}\left(\hat{x}_{k}\right) \\
\hat{x}_{k} & =z_{k}-E y_{k}
\end{aligned}
$$

where vector $z \in R^{n}$ and $\hat{x}_{k}$ is the estimation of $x_{k}$. The matrices $N, G, L, M$, and $E$ must be determined such that the error dynamics $e_{k}=\hat{x}_{k}-x_{k}$ converge to zero asymptotically. The following theorem gives the conditions for stability of dynamics $e_{k}$.

Theorem 1. For given scalar $\gamma$, if there exist real matrices $N$, $M$, and $P>0$ with appropriate dimensions such that inequality below to be valid,

$$
\left[\begin{array}{ccc}
-P+\gamma^{2} I & 0 & N^{T} P \\
0 & -I & D^{T} M^{T} P \\
P N & P M D & -P
\end{array}\right]<0
$$


then the state estimation error produced by observer (3) tends to zero asymptotically.

Proof. Refer to Delshad (2014).

To design the observer, it is necessary to find matrices $E, K$ and $P>0$ by any means such that inequality (4) is satisfied. To tackle this problem, we try to convert (4) to LMI and get a feasible solution through.

Theorem 2. For given scalar $\gamma$, if there exist real matrices $\bar{Y}$, and $P$ with appropriate dimensions such that LMI below to be valid,

$$
\left[\begin{array}{ccc}
-P+\gamma^{2} I & 0 & \delta_{13} \\
0 & -I & \delta_{23} \\
\delta_{13}^{T} & \delta_{23}^{T} & -P
\end{array}\right]<0
$$

where,

$$
\begin{aligned}
& \delta_{13}=A^{T} P+(U C A)^{T} P+(Y V C A)^{T} P-C^{T} \bar{K}, \\
& \delta_{23}=D^{T} P+(U C D)^{T} P+(Y V C A)^{T} P-(V C D)^{T} \bar{Y}
\end{aligned}
$$

$Y=P^{-1} \bar{Y}$, and $K=P^{-1} \bar{K}$, then the state estimation error produced by observer tends to zero asymptotically and the observer gains of (3) will be computed as:

$$
\begin{aligned}
E & =U+Y V \\
M & =I+E C \\
N & =M A-K C \\
L & =K(I+C E)-M A E
\end{aligned}
$$

Proof. Refer to Delshad (2014).

In summary, the full-order observer algorithm for the class of nonlinear systems under study is itemized as follows,

- Find suitable Lipschitz constant $\gamma$ satisfying Assumption 1

- Compute $U=-D_{1}\left(C D_{1}\right)^{+}$, and $V=I-\left(C D_{1}\right)\left(C D_{1}\right)^{+}$

- Solve the LMI defined by (5) for $\bar{Y}, \bar{K}$, and $P$

- Compute $Y=P^{-1} \bar{Y}$, and $K=P^{-1} \bar{K}$

- Using $Y$ and $K$, compute the observer gains as (7)

\section{CONSERVATIVENESS DISCUSSION}

Generally speaking, in case of any nonlinear Lipschitz function in the system dynamics there would be a conservativeness issue. Regarding observer design for instance, the issue is related to how big the Lipschitz constant can be to still have the stability of the error dynamics. Finding the maximum value of the admissible Lipschitz constant such that the observer performance is kept can be done via an optimization problem as follows.

Theorem 3. Consider the system (1) together with the nonlinear observer (3). Assume that there exist real matrices $\bar{Y}, \bar{K}$, and $P>0$ with appropriate dimensions, such that the following LMI optimization problem has a feasible solution:

$$
\text { s.t. } \quad\left[\begin{array}{cccc}
-P & 0 & \delta_{13} & I \\
0 & -I & \delta_{23} & 0 \\
\delta_{13}^{T} & \delta_{23}^{T} & -P & 0 \\
I & 0 & 0 & -\rho I
\end{array}\right]<0
$$

where,

$$
\begin{aligned}
& \delta_{13}=A^{T} P+(U C A)^{T} P+(Y V C A)^{T} P-C^{T} \bar{K}, \\
& \delta_{23}=D^{T} P+(U C D)^{T} P+(Y V C A)^{T} P-(V C D)^{T} \bar{Y}
\end{aligned}
$$

$Y=P^{-1} \bar{Y}$, and $K=P^{-1} \bar{K}$, then the state estimation error produced by observer (3) tends to zero asymptotically and the observer gains will be computed as:

$$
\begin{aligned}
E & =U+Y V \\
M & =I+E C \\
N & =M A-K C \\
L & =K(I+C E)-M A E
\end{aligned}
$$

the maximum bound of the $\gamma_{\max }$ can be calculated as,

$$
\gamma_{\max }=\sqrt{\frac{1}{\rho}}
$$

Proof. Refer to Delshad (2014).

\section{SIMULATION RESULTS AND DISCUSSIONS}

In the full version of this paper (as in Delshad (2014)), the proposed observer is first illustrated by a simulation example. We try to compare performance and conservativeness of our proposed method with other recent well-known researches in the literature within two subsections; performance discussion and conservativeness. In the simulation, we consider a singlelink manipulator with revolute joint as in Fig. 1.

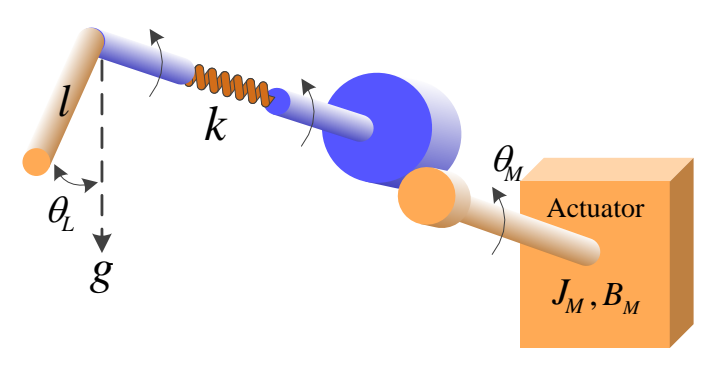

Fig. 1. Single-link flexible robot

A comparison between our proposed observer design technique and similar researches (such as Allgower (2008); Phanomchoeng (2010); Zemouche (2013)) will show the merits of the method in which our design exhibits better performance and less conservativeness compared to others.

\section{REFERENCES}

T. Raff, M. Kogel, and F. Allgower, "Observer with sampleand-hold updating for Lipschitz nonlinear systems with nonuniformly sampled measurements," American Control Conference, pp. 5254-5257, 2008.

G. Phanomchoeng, and R. Rajamani, "Observer Design for Lipschitz Nonlinear Systems Using Riccati Equations," American Control Conference, pp. 6060-6065 , 2010.

A. Zemouche, and M. Boutayeb, "On LMI conditions to design observers for Lipschitz nonlinear systems," Automatica, vol. 49, pp. 585591, 2013.

S. S. Delshad, and T. Gustafsson, "Observer Design for a Class of Nonlinear Systems Subject to Unknown Inputs", European Control Conference, Strasbourg, France, June 2427, 2014. (Accepted and will be presented) 